\title{
Videoendoscopia via posterior para cirurgia de hérnia de disco lombar
}

\author{
Luiz Henrique de Mattos Pimenta, Oswaldo I. Tella Jr., Ricardo Zanetti, Chester Sutterlin III
}

Serviço de Neurocirurgia, Hospital Santa Rita - São Paulo

North Florida Regional Medical Center - Gainesville, USA

\section{RESUMO}

Os autores apresentam a técnica de abordagem videoendoscópica posterior da coluna lombar em 63 casos operados com seguimento médio de 1 ano e 8 meses. A técnica videoendoscópica, via posterior, da coluna lombar, conhecida como MED (Microendoscopic Discectomy) foi idealizada com a finalidade de transformar a cirurgia convencional, já bem estabelecida e padronizada, em uma cirurgia minimamente invasiva sob os princípios da cirurgia videoendoscópica. Portanto, por essa via de acesso, podem-se realizar todos os procedimentos efetuados a céu aberto, tais como hemilaminectomia, facetectomia medial, foraminotomia, retração da raiz nervosa e a discectomia.

Os resultados iniciais em 63 cirurgias apontam para a tendência à recuperação mais rápida do paciente, que obtém alta hospitalar no mesmo dia da cirurgia e retorno ao trabalho em 15 dias, ou menos, em 50\% dos nossos casos. A incisão cirúrgica é bastante reduzida $(1,6 \mathrm{~cm})$. Não houve infecção em nossos casos. Como principais desvantagens, citamos o aumento do tempo cirúrgico e a dificuldade da técnica cirúrgica que impõe treinamento específico. Em um futuro próximo, será possível a realização dessa cirurgia em nível ambulatorial e sob anestesia local, permitindo, inclusive, a realização em pacientes idosos e de alto risco.

\section{PALAVRAS-CHAVE}

Hérnia discal lombar. Técnica cirúrgica. Videoendoscopia.

\section{ABSTRACT}

The microendoscopic discectomy. A new trend in the treatment of lumbar disc disease.

The authors report their experience with 63 cases of lumbar disc operated on through videoendoscopic technique. The microendoscopic discectomy (MED) was done by a posterior approach with fluoroscopic control through a paramedian $16 \mathrm{~mm}$ incision.

The initial results, with a mean follow up of 1 year and 8 months, point out to a short hospitalization time, early return to work and no case of infection. The main disadvantage is the prolonged time of surgery till you get familiar with the technique. This minimally invasive technique will allow in the near future that lumbar disc surgery will be performed under local anesthesia and as a dayclinic procedure.

\section{KEY WORDS}

Lumbar disc herniation. Spinal videoendoscopy. Surgical technique. 


\section{Introdução}

As técnicas cirúrgicas menos invasivas vêm sendo utilizadas em todas as áreas médicas. O acesso percutâneo para o tratamento da hérnia de disco lombar existe há mais de 30 anos, com a tentativa do uso de quimopapaína para a lise do disco ${ }^{18}$. Técnicas de nucleotomia percutânea usando instrumentos manuais foram descritas em $1975^{6}$. Essa técnica evoluiu nos anos seguintes para incluir o uso de aparelhos automatizados para a remoção do disco ${ }^{10,11}$, a endoscopia de coluna ${ }^{6} \mathrm{e}$ o $\operatorname{laser}^{2,16}$.

Embora bons resultados tenham sido publicados ${ }^{9,11}$, essas técnicas não se mostraram tão eficientes quanto a cirurgia a céu aberto para a hérnia de disco lombar. Mochida ${ }^{9}$ descreveu, em pacientes selecionados, 70\% de bons resultados com a nucleotomia percutânea. Onik ${ }^{10,11}$, com sua técnica de discectomia percutânea automatizada referiu bons resultados em $70 \%$ a $80 \%$ dos casos.

Entretanto, em estudos multicêntricos ${ }^{7}$, comprovouse que a discectomia percutânea possibilita retorno ao trabalho em apenas $55 \%$ dos pacientes e o mais importante é que as indicações para esse procedimento se limitavam às hérnias de disco contidas, sendo contraindicado nos casos de hérnia de disco com fragmento extruso e conseqüente déficit radicular.

Os avanços tecnológicos de óptica e instrumental permitiram o uso bem-sucedido de princípios cirúrgicos videoendoscópicos, menos invasivos, nas cirurgias da vesícula biliar, do joelho e do tórax. Nesses casos, a eficácia cirúrgica mostrou-se semelhante às técnicas convencionais, com a vantagem de proporcionar redução do período de internação hospitalar do paciente e retorno rápido ao trabalho.

Foley $^{3,5}$ descreveu, em 1997, a técnica denominada discectomia microendoscópica (MED), cujos princípios se baseiam em técnicas menos invasivas, endoscópicas, obedecendo a mesma via de acesso realizada pela técnica convencional, ou seja, por meio de hemilaminectomia, descomprimindo a raiz nervosa. Todos os passos cirúrgicos da técnica a céu aberto são, portanto, realizados por um afastador tubular (canal de trabalho), ao qual se acopla o endoscópio, com auxílio do intensificador de imagem. É possível, assim, realizarmos a hemilaminectomia, a retirada do ligamento amarelo, afastamento da raiz nervosa e retirada de fragmentos de discos extrusos ou a descompressão nos casos de estenose de canal medular.

Essa técnica abrevia a hospitalização do paciente, bem como seu retorno ao trabalho, em curto espaço de tempo ${ }^{3,13,14}$. A eficiência da técnica a longo prazo, entretanto, não pode ser avaliada no presente momento.

\section{Casuística e métodos}

São analisados 63 pacientes operados de hérnia de disco lombar pela videoendoscopia da coluna lombar via posterior. Os pacientes foram operados em dois serviços: 32 pacientes operados no North Florida Regional Medical Center, Gainesville, Flórida, a partir de dezembro de 1996, e 31 pacientes operados no Hospital Santa Rita, São Paulo, a partir de setembro de 1997.

\section{Técnica cirúrgica}

A técnica cirúrgica empregada para discectomia microendoscópica foi, de maneira sucinta, a descrita abaixo (Figs. 1 a 12):

1) Posicionamento: o paciente, sob anestesia geral, peridural ou local, em decúbito ventral, é posicionado sobre um suporte metálico, conhecido como "País", que permite boa abertura do espaço interlaminar, ao mesmo tempo que garante o abdome bem livre. $\mathrm{O}$ cirurgião (destro) posiciona-se à direita do paciente, tendo a instrumentadora, eventuais auxiliares, vídeo e o monitor do intensificador de imagem posicionados à esquerda do paciente. Note-se que o cirurgião trabalha ao lado do intensificador.

2) Incisão: o ponto de entrada da incisão deve ser feito $1,5 \mathrm{~cm}$ a $2 \mathrm{~cm}$ lateralmente à linha média, no nível do espaço discal. Sob fluoroscopia, insere-se o mandril que deve ser dirigido ao meio do disco patológico. É realizada, então, a incisão paramediana de mais ou menos $1,6 \mathrm{~cm}$.

3) Dilatadores: a introdução do primeiro dilatador sob visão fluoroscópica é fundamental, pois este precisa atingir o bordo inferior da lâmina superior, entre o processo espinhoso e a faceta articular. Essa ação consegue-se facilmente quando o tacto vai ficando mais apurado. Seguem-se a colocação dos demais dilatadores e, finalmente, do afastador tubular que é fixado no braço flexível (conhecido como "Leilão"), ficando completamente imóvel e em contato com a lâmina.

4) Colocação do endoscópio: o componente óptico do endoscópio, uma fibra de $2 \mathrm{~mm}$ de diâmetro, $10 \mathrm{~cm}$ de comprimento e angulada em $25^{\circ}$, é introduzido no afastador tubular (que é o "canal de trabalho") e fica fixo em sua parede interna, podendo ser "rodado" de forma a permitir uma visão de $360^{\circ}$. O sistema óptico pode descer ou subir no tubo e produz uma 
magnificação tanto maior quanto mais próxima do tecido, chegando a aumentos de até 50 vezes. A ponta do endoscópio pode ficar embaçada ou engordurada, sendo necessário sua limpeza.

5) Orientação da imagem: ao introduzir o endoscópio, a imagem circunferencial no monitor pode não corresponder à anatomia do campo cirúrgico. Introduz-se, então, um instrumental em um determinado ponto; por exemplo, coloca-se um dissector no ponto inferior do campo que corresponderia às 6 horas de um relógio e roda-se o acoplador da câmera, rodando a imagem do monitor, produzindo a equivalência das imagens do monitor com a anatomia do campo cirúrgico.

6) Identificação da lâmina: identifica-se a lâmina com uma cureta e retira-se todo o tecido mole (fibras e músculos) localizado sobre esta. Consegue-se isso com bisturi elétrico. Destacase o ligamento amarelo da lâmina, com o auxílio da cureta.

7) Hemilaminectomia e facetectomia: são utilizadas goivas de Kerrison próprias para esses procedimentos, ou com auxílio do drill (principalmente nos casos de estenose de canal).

8) Ressecção do ligamento amarelo: abre-se o ligamento amarelo com a introdução da cureta sob a lâmina, "descolando" o ligamento da face inferior da lâmina. Retira-se o resto de ligamento com goiva de Kerrison.

9) Exposição da raiz e identificação do disco: nesse tempo, identifica-se a raiz que apresenta brilho azulado, característico da dura-máter. $\mathrm{O}$ afastamento da raiz, com dissector ou com aspirador delicado, permite a identificação do disco. Pode-se explorar o espaço sob a raiz com o dissector angulado de "bola". Veias epidurais podem ser coaguladas com o coagulador bipolar.

10) Abertura do disco e remoção: a abertura do anel fibroso do disco faz-se com microbisturi, após a proteção da raiz com afastador. Retirase o disco com pinças de Love, da mesma maneira que se faz a céu aberto. Se necessário, introduz-se a cureta invertida que serve também para "compactar" o conteúdo discal facilitando a posterior remoção. Pode-se realizar uma foraminotomia se quiser ampliar o espaço da raiz.

11) Fechamento: o fechamento se faz com um ou dois pontos de fio absorvível na fáscia e sutura intradérmica sem fios aparentes. Coloca-se apenas um band-aid como curativo.

\section{Resultados}

A idade dos pacientes variou de 19 a 87 anos, com média de 47,4 anos; 7 pacientes tinham idade entre 70 e 87 anos ( $11 \%$ dos casos).

Quanto à distribuição de sexo, 33 pacientes eram do sexo feminino e 30 do sexo masculino. Quanto à profissão, observamos que, entre os 63 pacientes operados, 17 eram trabalhadores braçais (em que o esforço físico faz parte da profissão).

Todos os pacientes operados eram portadores de quadro de compressão radicular. Apenas $9 \%$ dos pacientes não tinham déficit sensitivo ou motor, sendo a indicação do tratamento a dor ciática rebelde, associada aos resultados da tomografia ou ressonância e falha dos tratamentos conservadores.

Quanto ao ato cirúrgico, avaliados os tempos, notamos que a duração do procedimento variou de 70 a 240 minutos, com média de 120 minutos. Entre os casos norte-americanos, a média foi de 110 minutos, enquanto os casos brasileiros levaram, em média, 137 minutos.

A reversão de técnica endoscópica para céu aberto ocorreu em 5 casos: 2 casos americanos e 3 casos brasileiros. Em 4 destes, houve a suspeita de fístula liquórica que só se confirmou em 2 casos que tiveram a dura-máter suturada. Dois casos tratavam-se de reoperação.

Quanto ao nível operado, em 36 pacientes foi em L5-S1, 23 em L4-L5, 3 em L3-L4, e 1 em L1-L2.

Alta hospitalar foi dada no mesmo dia da cirurgia, mais ou menos 8 horas após o final da mesma, em 56 pacientes $(89 \%)$.

$\mathrm{O}$ resultado do procedimento foi dividido em mau, regular, bom ou ótimo de forma grosseira. Obtivemos, em 58 pacientes, resultado bom ou ótimo. Em 2 casos foi necessária reoperação por ter ficado fragmento discal.

Todos os pacientes, menos um, foram submetidos à anestesia geral. Em uma paciente de 87 anos, de alto risco segundo o cardiologista, o procedimento foi realizado sob anestesia local (marcaína 0,5\%).

Retorno ao trabalho aconteceu em $80,6 \%$ dos casos brasileiros ( 25 pacientes). O retorno ocorreu após 24,6 dias, em média; os trabalhadores braçais retornaram ao trabalho, em média, após 34,7 dias e, os demais, após 18 dias.

\section{Discussão}

A introdução da técnica denominada Microendoscopic Discectomy (MED) deve-se a Kevin T. Foley, em 1996, com a finalidade de tornar a cirurgia da hérnia 


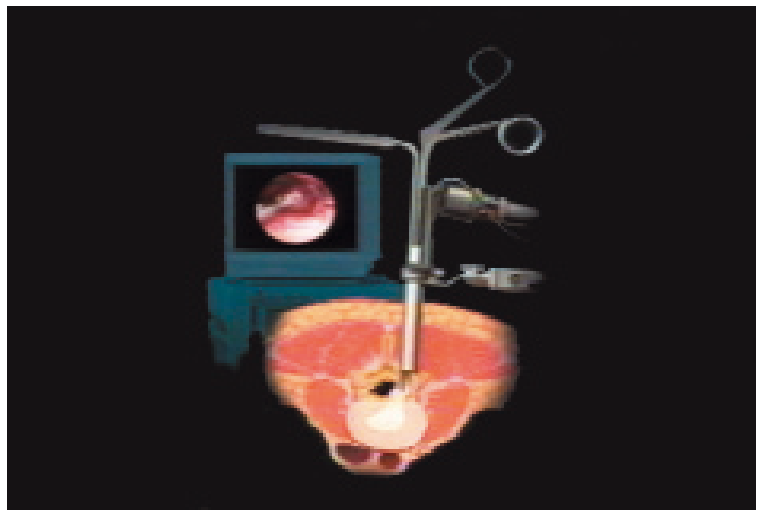

Figura 1 - Desenho esquemático da técnica da videoendoscopia.

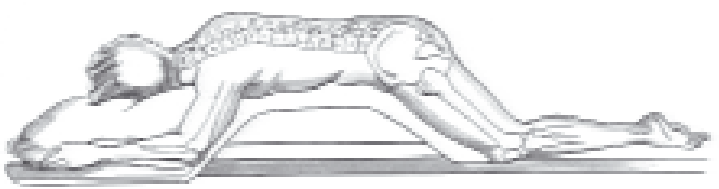

Figura 2 - Posição do paciente no suporte.

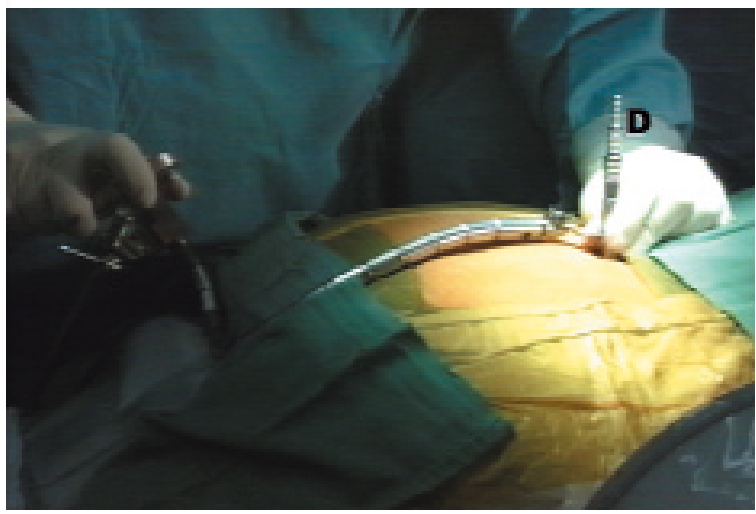

Figura 3 - Colocação dos dilatadores e fixadores com "Leilão".

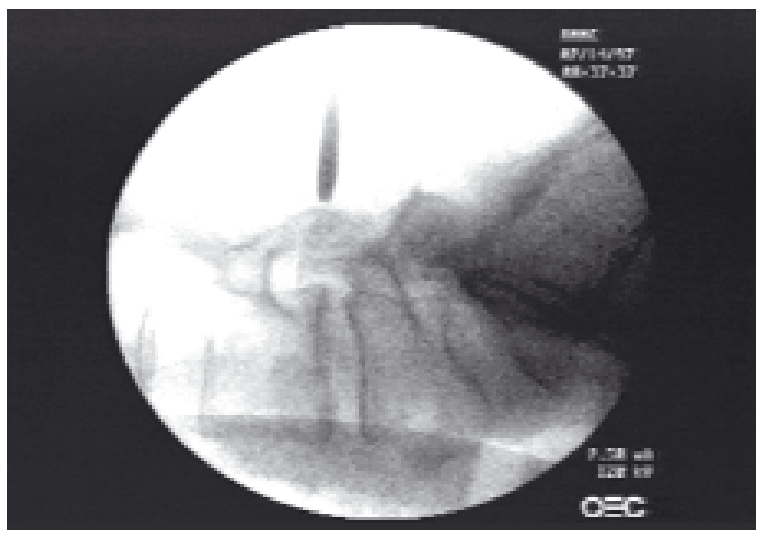

Figura 4 - Controle radioscópico da direção do disco.

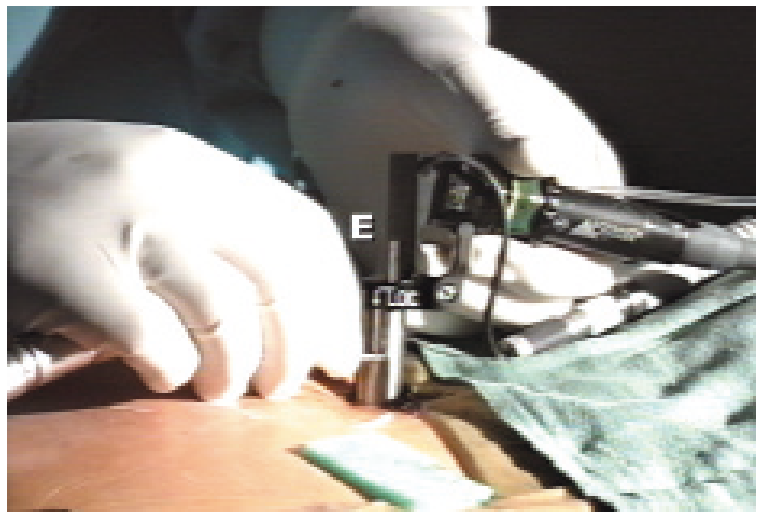

Figura 5 - Colocação do endoscópio no canal de trabalho.

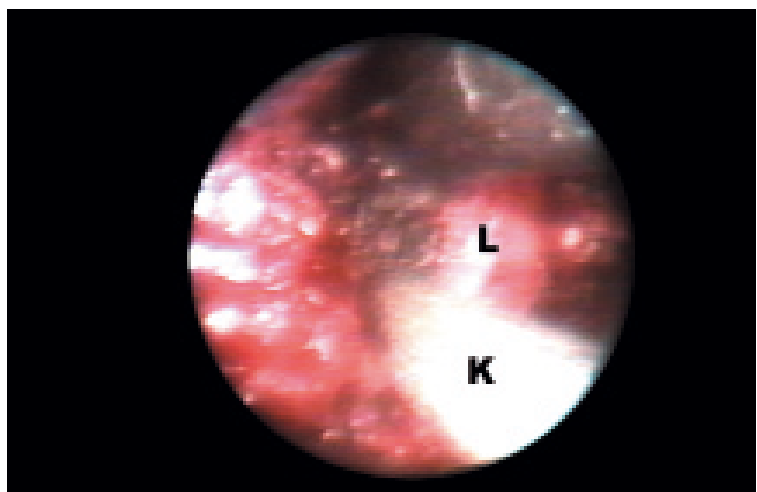

Figura 6 - Hemilaminectomia $-K=$ Kerrison $L=$ Lâmina.

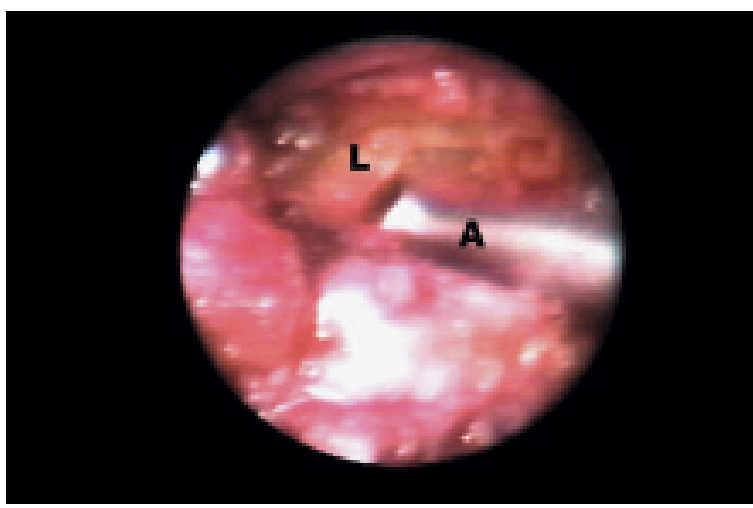

Figura 7 - Ligamento amarelo $(L)$ mostrado com aspirador $(A)$

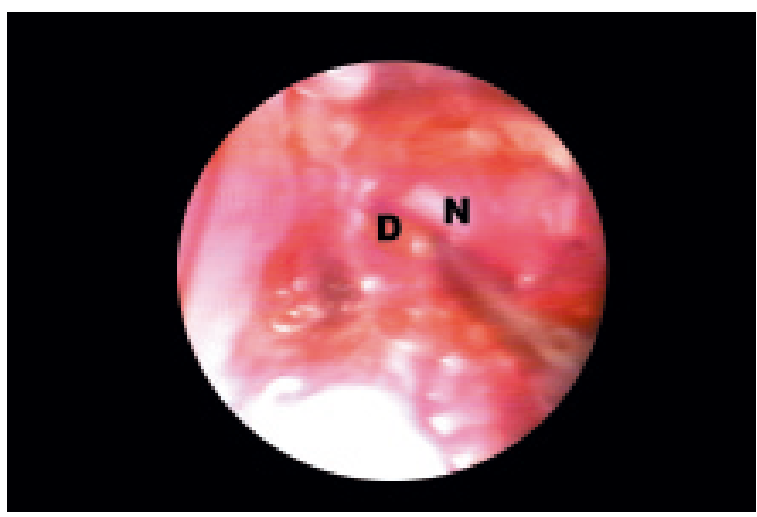

Figura 8 - Indentificação da raiz $(N)$ e hérnia de disco (D). 


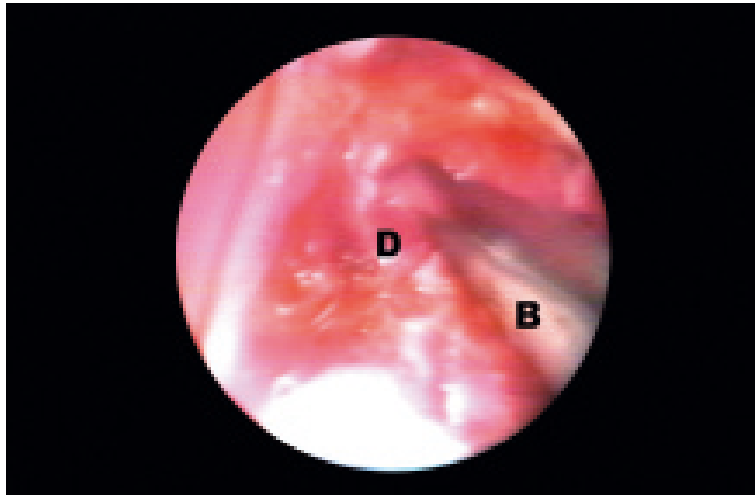

Figura 9 - Incisão do disco (D) com bisturi (B).

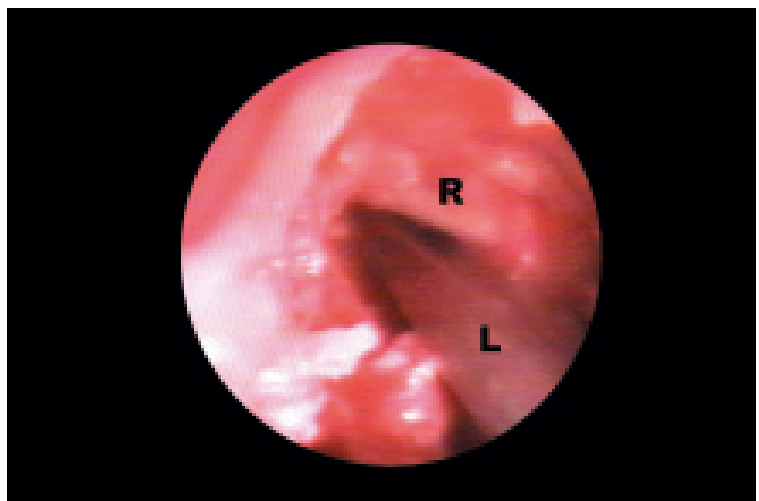

Figura 10 - Introdução pinça de Love (L) ao lado da raiz (R).

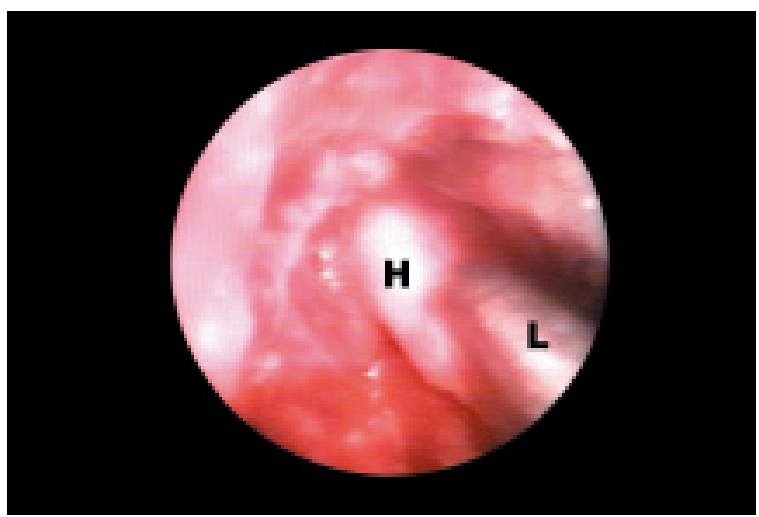

Figura 11 - Retirada da hérnia de disco $(H)$ com a pinça de Love.

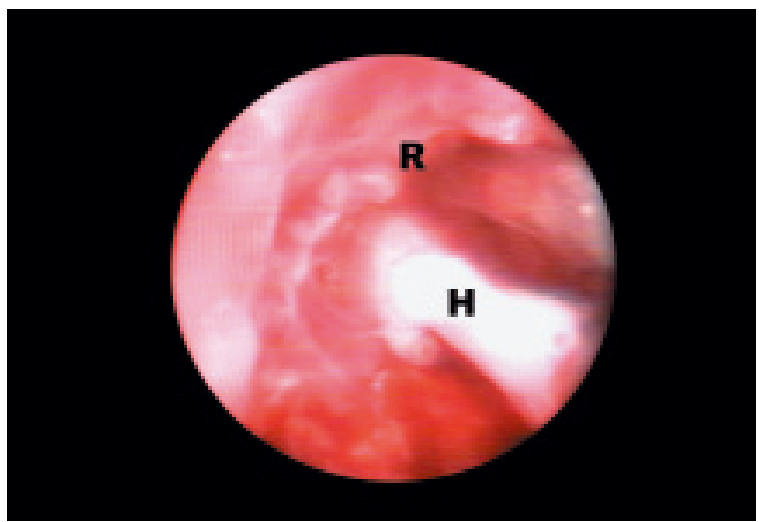

Figura 12 - Fragmento de disco $(H)$ saindo ao lado da raiz (R). de disco, já padronizada, menos invasiva. O uso de sistemas ópticos e instrumentos mais sofisticados, bem como o uso bem sucedido da videoendoscopia em cirurgias de vesícula, joelho e tórax, incentivaram a aplicação dos mesmos para a cirurgia da hérnia discal lombar. Até então, as técnicas endoscópicas para a cirurgia da hérnia de disco lombar tinham sua indicação limitada às hérnias contidas.

A videoendoscopia com abordagem posterior da coluna, seguindo os mesmos passos da cirurgia (com microscópio) já padronizados, permitiria a retirada de hérnias extrusas e migradas, cujas indicações são as melhores.

A casuística de Folley e $\mathrm{Smith}^{3}$ compreende 150 pacientes submetidos a MED para hérnias discais lombares e estenose de canal (12\%). As indicações cirúrgicas fogem ao escopo desse trabalho.

Outro trabalho apresentou estudo cooperativo abrangendo 100 pacientes operados por quatro cirurgiões de coluna de diferentes locais dos Estados Unidos ${ }^{13}$. Um terceiro trabalho, igualmente cooperativo, analisou 88 pacientes submetidos ao MED entre cinco grupos de cirurgiões ${ }^{14}$.

Portanto, o número de pacientes ainda é pequeno em todas as estatísticas e o seguimento ainda é curto.

Nosso material de 63 casos inclui pacientes operados em Gainesville (32 casos) e em São Paulo (31 casos).

As distribuições etária e de sexo assemelham-se às da literatura mundial. Podemos apenas ressaltar o número importante de pacientes idosos que, com técnica menos invasiva, podem ser operados com redução de riscos. $\mathrm{Na}$ casuística de Folley ${ }^{4}$, a idade variou de 23 a 67 anos. Em 61\% dos pacientes havia fragmento de disco expulso. Não obtivemos essa avaliação em nosso material. O estudo cooperativo de Albuquerque $^{13}$ mostrou que a média de duração do procedimento operatório é $10 \%$ maior na técnica videoendoscópica (media de 100,9 minutos). Em nossa casuística, a média foi de 120 minutos.

$\mathrm{O}$ índice de fístula liquórica no material de Folley ${ }^{5}$ foi de 2,5\%, enquanto no estudo cooperativo foi de $5,1 \%{ }^{13}$. Em nossa casuística, tivemos $7,5 \%$ de casos com suspeita de fístula (revertidas para técnica a céu aberto), mas em apenas 4,5\% foram confirmadas e tratadas. Tivemos, ainda, um caso de disestesia pósoperatória no trajeto do ciático, com persistência por 30 dias, decorrente de provável tração excessiva da raiz (um de nossos casos iniciais). Não encontramos essa complicação descrita.

O tempo de hospitalização foi de 8 horas em $89 \%$ de nossos casos. O estudo cooperativo reporta o tempo variando de 2 a 48 horas $^{13}$. Nos casos de Folley ${ }^{4}$, a média de internação foi de 6 horas. Quanto ao resultado 
clínico, Folley ${ }^{13}$ referiu que todos seus pacientes, menos um, tiveram resultado bom ou ótimo. O estudo cooperativo cita a redução de sangramento pela técnica videoendoscópica. Não estudamos esse aspecto no nosso material ${ }^{13}$.

Folley e Smith ${ }^{4}$ preferem o uso da anestesia peridural em seus pacientes. Não descreveram o uso de anestesia local, embora citem essa possibilidade. No estudo cooperativo, utilizou-se anestesia geral ${ }^{14}$. Em nossos pacientes, utilizamos anestesia geral com exceção de uma paciente de 87 anos com contraindicação formal para anestesia geral, em que realizamos a cirurgia apenas com uso local de marcaína a $0,5 \%$.

Em nossa casuística, obtivemos resultado bom ou ótimo em 58 dos 63 pacientes. Em 2 casos foi necessária reoperação, após 90 dias, por ter ficado fragmento de disco.

Retorno ao trabalho, segundo Folley ${ }^{4}$, foi possível após 14 dias entre pacientes trabalhadores não-braçais e, após 58 dias, entre trabalhadores braçais. No estudo cooperativo, $65,8 \%$ dos pacientes voltaram ao trabalho, em média global, após 21,5 dias ${ }^{13}$. Em nosso material, entre os 31 pacientes operados no Hospital Santa Rita, $25(80,6 \%)$ retornaram ao trabalho. Em média, esse retorno ocorreu em 24,6 dias. Se computados apenas os pacientes com profissão do tipo trabalhador braçal (10 pacientes), o tempo médio para retorno ao trabalho foi de 34,7 dias, enquanto no grupo de profissionais não-braçais (15 pacientes), foi de 18 dias.

\section{Conclusões}

A técnica videoendoscópica para o tratamento da hérnia de disco lombar é minimamente invasiva e tornase simples após treinamento. Permite a retirada de grandes hérnias extrusas, com menor lesão dos tecidos. Possibilita a recuperação mais rápida do paciente, ou seja, diminui o tempo de internação hospitalar (menos de um dia) e o retorno ao trabalho é igualmente precoce. O tamanho da incisão é, em média, de $1,6 \mathrm{~cm}$. Com essa técnica, é possível operar pacientes idosos com anestesia local.

Não se pode ainda concluir sobre os resultados a longo prazo, embora se possa supor que haja uma redução dos casos de fibrose epidural pós-operatória, uma vez que a manipulação desse espaço é muito menor.

\section{Referências}

1. APOSTOLIDES PJ, JACOBOWITS R, SONNOTAG VK Lumbar discectomy microdiscectomy: the gold standard. Clin Neurosurg 43:228-38, 1978.

2. CHOY DSJ, CASE RB, FIELDING W: Percutaneous laser nucleolyses of lumbar disks. N Engl J Med 317:771-2, 1987.

3. FOLLEY K: Personal comunication.

4. FOLLEY K, SMITH MM: Microendoscopic discectomy. Techniq Neurosurg 3:301-7, 1997.

5. FOLLEY K, SMITH MM: Microendoscopic discectomy (MED): surgical technique and initial clinical results. Presented at the North American Spine Society 12th Annual Meeting, New York, October, 1997. Abstract.

6. HIJIKATA S, YAMAGISHIM: Percutaneous discectomy: a new treatment method for lumbar disc herniation. J Toden Hosp 5:5-13, 1975.

7. KAHANOVITZ N VIOLA K: A multicenter analysis of percutaneous discectomy. Spine, 15:713-5, 1990.

8. MAROON JC, QUIGLEY MR, GLEASON PL: Is there a future for percutaneous intradiscal therapy? Clin Neurosurg 43:239-51, 1996.

9. MOCHIDA J, ARIMA T: Percutaneous nucleotomy in lumbar disc herniation. A prospective study. Spine 18:2063-8, 1993.

10. ONIK G, HELMS CA: Percutaneous lumbar discectomy using a new aspiration probe. AJR 144:1137-40, 1985.

11. ONIK G, MOONEY V, MAROON, JC: Automated percutaneous discectomy: a prospective multiinstitucional study. Neurosurgery 26:228-33, 1990.

12. PROPST D, SUTTERLIN III C: Microendoscopic discectomy system vs microdiscectomy. North Florida Regional Medical Center, Gainesville Report, 1997.

13. PHDX: MED System Outcomes Assesment Data. December 1997.

14. PHDX: MED System Outcomes November, 1997

15. QUIGLEY MR, MAROON JC: Automated percutaneous discectomy. Neurosurg Clin N Am 7:29-35, 1995.

16. QUIGLEY MR: Percutaneous laser discectomy. Neurosurg Clin N Am. 7:37-42, 1996.

17. SHAPIRO S: Long-term follow-up of 57 patients undergoing automated percutaneous discectomy. J Neurosurg 83:31-3, 1995.

18. SCHREIBER A, SUEZAWA Y: Transdiscoscopic percutaneous nucleotomy in disk herniation. Orthop Rev 15:35-8, 1986

19. SMITH L: Enzyme dissolution of the nucleus pulposus in humans. JAMA 265:137-40, 1964.

20. SUTTERLIN CE: Microendoscopic Discectomy. In: Open and Minimaly Invasive Surgery of the Lumbar Spine, Memphis Feb 27-8, 1998.

21. ZELKO J R, MISKO J, SWANSTROM L, PENNINGS J, KENVON T: Laparoscopic lumbar discectomy. Am J Surg 169:496-8, 1995.

Original recebido em julho de 1998

Aceito para publicação em janeiro de 1999

\section{Endereço para correspondência}

Luiz Henrique de Mattos Pimenta

Rua Borges Lagoa, 783/61

CEP 04038-031 - Vila Clementino, São Paulo, SP 